\title{
Schlegels Republik und Ungers Offizin
}

\author{
Die politische Frühromantik im Kontext \\ ihrer Publikationsmedien
}

I.

Zweifelsohne gehört Friedrich Schlegels Aufsatz Versuch über den Begriff des Republikanismus veranlaßt durch die kantische Schrift zum ewigen Frieden zu den Klassikern der politischen Romantik. Er war 1796 in Johann Friedrich Reichardts Journal Deutschland erschienen und ist von der Forschung bisher vornehmlich unter philosophiegeschichtlichen, ${ }^{\mathrm{I}}$ (kultur)semiotischen ${ }^{2}$ und werkhistorischen ${ }^{3}$ Gesichtspunkten behandelt worden. Konsens ist hier, dass Schlegel im Grunde keine Rezension der kantischen Schrift, sondern vielmehr eine Abhandlung eigenen Rechts vorgelegt hat, die als Symptom seiner Auseinandersetzung mit der Französischen Revolution zu deuten sei. Unstreitig ist auch, dass Schlegel

I Klaus Peter: „Einleitung«. In: ders.: Die politische Romantik in Deutschland. Eine Textsammlung. Stuttgart 1985, S. 9-73; Friederike Rese: „Republikanismus, Geselligkeit und Bildung. Zu Friedrich Schlegels Versuch über den Begriff des Republikanismus«. In: Athenäum 7 (1997), S. 37-71; Jürgen Stahl: "Die Idee des sewigen Friedens in der bürgerlich-demokratischen Publizistik Friedrich Schlegels und Joseph Görres«. In: Bolko Schweinitz (Hg.): Philosophie und Frieden. Beiträge zum Friedensgedanken in der deutschen Klassik. Weimar 1985 , S. 155-169.

2 Peter Schnyder: "Politik und Sprache in der Frühromantik. Zu Friedrich Schlegels Rezeption der Französischen Revolution«. In: Athenäum 9 (1999), S. 39-65; Franz-Josef Deiters: "Die Poesie ist eine republikanische Reder - Friedrich Schlegels Konzept einer selbstreferentiellen Dichtung als Vollendung der Politischen Philosophie der europäischen Aufklärung«. In: DVjs 8I (2007), S. 3-20.

3 Peter D. Krause: "'Vollkommene Republikı. Friedrich Schlegels frühe politische Romantik«. In: IASL 27 (2002), S. I-3I. 
Überlegungen aus dem Studium-Aufsatz mittels philosophischer Methodik fortführen will. Er zeigt sich interessiert an einer $>$ Deduktion` der Begriffe Freiheit und Gleichheit, deren Begründung Kant in dessen Schrift nicht gelungen sei. Für Schlegel ist die Deduktion der Begriffe allerdings unabdingbare Voraussetzung für die Auffindung einer institutionalisierten Friedensordnung, wie sie Kant vorschwebt.

Fragt man nach den realpolitischen Konsequenzen, die aus den im Republikanismus-Aufsatz erzielten Differenzierungen hätten erwachsen können, so gehen die Beurteilungen indes auseinander: Schlegels Affinität zu ästhetischen Schreibweisen und "poetischen Verinnerlichungskonzepten $\aleph^{4}$ habe unausweichlich eine "Unterbietung des gesellschaftlichen Zusammenhangs " 5 sowie "eine vollständige Derealisation des Wirklichen ${ }^{6}{ }^{2}$ zur Folge gehabt. Peter D. Krause, der auf solchen Urteilen den langen Schatten Carl Schmitts ruhen sieht, betont hingegen, dass Schlegel gerade die "[p]oetische Entgrenzung als Ermöglichung des Politischen " 7 ansehe. Ein triftiges Gegenargument ist damit nicht gefunden, denn dass dies prinzipiell möglich sei, bestreiten gerade jene Forscher, die Schlegel vorwerfen, die Kunst "von den empirischen Lebensverhältnissen ${ }^{8}$ abgetrennt zu haben.

Wie lässt sich hier vermitteln? Zunächst, so möchte ich vorschlagen, durch eine methodische Umorientierung. Denn dem

4 Karl-Heinz Bohrer: Der romantische Brief. Die Entstehung romantischer Subjektivität. Frankfurt a. M. 1987, S. 49.

5 Bernd Bräutigam: „Eine schöne Republik. Friedrich Schlegels Republikanismus im Spiegel des Studium-Aufsatzes". In: Euphorion 70 (1976), S. 315-339, hier S. 333 .

6 Raimar Zons: „Das Schöne soll sein«. In: Ernst Behler/Jochen Hörisch (Hg.): Die Aktualität der Frühromantik. Paderborn u.a. 1987, S. 208-218, hier S. 208.

7 Krause: »Friedrich Schlegels frühe politische Romantik« (s. Anm. 3), S. 25. Ähnlich auch Deiters: "Die Poesie ist eine republikanische Rede«" (s. Anm. 2).

8 Ingrid Oesterle: "Der sglückliche Anstoß ästhetischer Revolution und die Anstößigkeit politischer Revolution. Ein Denk- und Belegversuch zum Zusammenhang von politischer Formveränderung und kultureller Revolution im Studium-Aufsatz Friedrich Schlegels«. In: Dieter Bänsch (Hg.): Zur Modernität der Romantik. Stuttgart 1977, S. 167-216, hier S. 202. 
ein allenfalls marginales Interesse entgegengebracht. Das mag verwundern. Denn nicht nur lässt sich generell konstatieren, dass politische Ideen Verbreitung finden müssen - wie sollten sie sonst "die Kapazität für kollektiv bindende Entscheidungen" je erzeugen, geschweige denn »bereithalten « ${ }^{9}$ können? Auch und vor allem die romantische Kulturpolitik ist wesentlich durch ihre Bereitschaft gekennzeichnet, subjektphilosophisch-ästhetische in soziale Programme zu übersetzen. ${ }^{\text {I }}$ Die Belastbarkeit dieser Hypothese lässt sich am Material leicht bestätigen, etwa durch einen Blick in den Republikanismus-Aufsatz. Wo Fichte formuliert "Der Mensch soll seyn ", ${ }^{\text {II }}$ heißt es bei Friedrich Schlegel: "Gemeinschaft der Menschheit soll sein, oder das Ich soll mitgeteilt werden. $\aleph^{\text {I2 }}$ Dieser frühromantische Impuls klingt noch nach in Heinrich Heines ironisch getöntem Satz, dass die "république des lettres" als »Gedankengütergemeinschaft "13 $^{13}$ aufzufassen sei.

Es kann bei einer Re-Deskription des Republikanismus-Aufsatzes aber nicht darum gehen, bloß ideengeschichtlich zu argumentieren. Denn dann liefe man erneut sehenden Auges in die Falle, die Carl Schmitt im Jahr I9I9 aufgestellt hat. Der Jurist hatte ja behauptet, dass die notorische, wirklichkeitsmeidende Unentschiedenheit der Romantiker, ihr »subjektivierter Occasionalismus « ${ }^{\mathrm{I4}}$ darin ihren Ausdruck gefunden habe, dass die Politik den Romantikern ein Gegenstand unter vielen war, um sich ästhetisch zu gebärden. Die

9 Niklas Luhmann: Die Politik der Gesellschaft. Hg. von André Kieserling. Frankfurt a. M. 2002, S. 26.

Io Vgl. Till Dembeck: »Kulturpolitik und Totalitarismus. Zur deutschen Romantik«. In: Merkur 66 (2012), S. I70-176.

II Johann Gottlieb Fichte: "Über die Bestimmung des Gelehrten«. In: ders.: Werke. Bd. 6. Hg. von Immanuel Hermann Fichte. Berlin I97I, S. 29I-346, hier S. 296.

I2 Friedrich Schlegel: »Versuch über den Begriff des Republikanismus veranlaßt [sic] durch die Kantische Schrift zum ewigen Frieden«. In: Deutschland. 3. Bd., 7. Stk. Berlin 1796, S. I0-4I, hier S. I8.

I3 Heinrich Heine: »Prosanotizen II (Mai I83I bis I848)«. In: ders.: Historischkritische Gesamtausgabe der Werke. Hg. von Manfred Windfuhr. Bd. Io: Shakespeares Mädchen und Frauen/Kleinere literaturkritische Schriften. Hg. von Jan-Christoph Hauschild. Hamburg 1993, S. 314-342, hier S. 32I.

I4 Carl Schmitt: Politische Romantik. 6. Aufl. Berlin 1998 [I919], S. I8. 
252 Folge: eine elaborierte, gleichwohl aber sentimental überspannte Kunst, die ihre repräsentative Potenz eingebüßt und sich in reflexiven Schleifen totgelaufen hat. Schmitt im O-Ton:

Denn was bedeutet sozial für die Kunst seit der Romantik? Entweder endete sie im s'art pour l'art‘, in der Polarität von Snobismus und Bohème, oder sie wurde zu einer Angelegenheit privater Kunstproduzenten für privatim interessierte Kunstkonsumenten. ${ }^{\text {Is }}$

Es tangiert daher den Kern der schmittschen Argumentation nicht, wenn man seine vermeintlich schmale Materialbasis angreift und dann auf andere fiktionale Texte verweist. ${ }^{16}$ Zum Prüfstein für die hier gegen Schmitt in Stellung gebrachte Behauptung, dass romantische Ideen sich in der realen Realität festgesetzt haben und soziale Folgen zeitigten, würde erst der umgekehrte Fall eines Transfers aus der Literatur in die Sphäre einer anderen Institution. ${ }^{17}$

Um diese auf die kulturpolitischen Potenziale der Frühromantik abstellende Perspektive einnehmen zu können, braucht es die Beobachtung des Publikationskontextes, den ich im Folgenden mit Gérard Genette als Paratext adressieren will. ${ }^{18}$ In den Blick geraten dann - als Peritext - jene verbalsprachlich-gedruckten Texte, die in unmittelbarer Nähe zum Republikanismus-Aufsatz angesiedelt sind, wie auch - als Epitext - solche, die in größerer Entfernung zu ihm zirkulieren. Im Falle des Peritextes werde ich mich für die Titel- und Mottoarrangements der Zeitschrift Deutschland interessieren, im Falle des Epitextes die Organisationseinheit in den Blick nehmen, die den Druck, den Verlag und schließlich die Versendung des Journals besorgt hat: Johann

I5 Ebd., S. I7.

I6 Vgl. Markus Schwering: "Politische Romantik«. In: Helmut Schanze (Hg.): Romantik-Handbuch. 2., durchges. u. aktual. Aufl. Tübingen 2003, S. 479-509, hier S. 479-48I.

I7 Zur Unterscheidung fiktionale Realität/reale Realität vgl. Niklas Luhmann: "Literatur als fiktionale Realität«. In: ders.: Schriften zu Kunst und Literatur. Hg. von Niels Werber. Frankfurt a. M. 2008, S. 276-29I.

I8 Nach Gérard Genette: Paratexte. Das Buch vom Beiwerk des Buches. Übers. v. Dieter Hornig. Frankfurt a. M. I989. 

formt die romantische Literatur jene Medien und Institutionen, durch die sie selbst hervorgebracht wird?

Blickt man auf das editoriale Setting des Journals, dann fällt sogleich der thematische Fokus ins Auge, den schon der Titel kommuniziert. Reichardts Zeitschrift will sich "mit Deutschland beschäftigen, und auf wahren Gemeinsinn, der uns guten Deutschen mehr als alles andere fehlt, abzwecken. $«$ '19 Das Projekt zielt also auf die Bildung einer kommunikativ verfassten Korporation, die den Namen Deutschland tragen wird und sich zu diesem Zweck ihrer selbst erinnern muss. Denn Deutschland I796: das ist eben eine fiktionale Realität; Intellektuelle, die an politischer Realisation interessiert sind, können bloß auf die Durchsetzungskraft des Mediums Zeitschrift vertrauen. Um sie zu stärken, greifen die Herausgeber für alle Hefte auf ein Motto aus der Feder Immanuel Kants zurück.

Fiat justitia, pereat mundus!

Es herrsche Gerechtigkeit, die Schelme der Welt mögen auch insgesamt darüber zu Grunde gehen!

Kant. Zum ewigen Frieden

Dieses Motto etabliert ein translatives und ein agonales Dispositiv, das einen Großteil der im Journal publizierten Texte durchzieht und nachgerade steuert. Translativ, weil die deutsche Nation als Produkt von Übersetzungen erfahrbar wird: ein Grundsatz des römischen Rechts, den jeder Leser der Zeitschrift ohnehin verstanden hätte, wird zusammen mit einem Satz Immanuel Kants präsentiert, der als erweiterte oder sehr freie Übersetzung zu

I9 [Johann Friedrich Reichardt:] "[Editorial]«. In: Deutschland. I. Bd., I. Stk. Berlin I796, S. I-6, hier S. I. 
254 beschreiben wäre. ${ }^{20}$ Von `Schelmen der Welt ist im Originalzitat keine Rede. Diese Hinzufügung markiert das agonale Dispositiv. Man wird damit rechnen können, dass die Reklamation spezifisch deutscher Kulturgüter mit der Herabsetzung anderer Nationen korreliert wird. Dies zeigt sich auch in den heftspezifischen Motti, die in eine Art Dialog mit dem Titel der Zeitschrift treten. Für das dritte Stück bspw. sieht das so aus: „D e u t s ch l a n d / Erkenne was du bist. - / Göthe. «Im siebten Stück des ersten Bandes - hier erscheint Schlegels Aufsatz - liest sich das heftspezifische Mottoarrangement wie folgt: "Deutschland / Wir verkennen nur die andern höflich, / damit sie wieder uns verkennen sollen - / Göthe.« Vom Dramenkontext befreit, kann Tasso für Reichardts Journal sprechen. Auch der andere Klassiker kommt zu seinem Recht. Schiller wird von Reichardt für das Motto des fünften Stückes des zweiten Bands mit den Worten zitiert: »Dem Verdienste seine Kronen, / Untergang der Lügenbrut!« Weimar antwortet auf die Provokationen. Das Xenion mit dem Titel Mottos ist ad personam Johann Friedrich Reichardt formuliert: »Setze nur immer Mottos auf deine Journale, sie zeigen / Alle die Tugenden an, die man an dir nicht bemerkt. $\ll^{21}$ Deutlich wird somit, dass Reichardt im literarischen Feld der I79oer Jahre als Herausgeber identifiziert ist, der sich durch Mottoarrangements zu positionieren versucht, wofür er denn auch attackiert wird. Der agonale Reiz, den der Kant-Spruch gesetzt hat, ist nicht ohne Reaktion geblieben.

Zudem verdeutlicht die Mottopolitik, dass Kants Schrift Zum ewigen Frieden eine eminente Bedeutung für das gesamte Journal zukommt. Wenn sich Schlegels Aufsatz also im Untertitel als veranlaßt durch die kantische Schrift zum ewigen Frieden zu erkennen gibt, dann ist davon auszugehen, dass sie sich zu den etablierten Dispositiven positionieren wird. Schlegels Text tritt denn auch tatsächlich in Korrespondenz zu einer Rezension der Schrift zum

20 Im Originaltext wird der Eindruck freilich noch dadurch verstärkt, dass der lateinische Satz den Zeitkonventionen entsprechend in Antiqua, der deutschsprachige aber in Ungers dezidiert 'deutscher Reformtypographie gesetzt ist.

2I Friedrich Schiller: Werke. Nationalausgabe. Bd. I: Gedichte in der Reihenfolge ihres Erscheinens I776-I799. Hg. von Julius Petersen u. Friedrich Beißner. Weimar 1943, S. 336. 
ewigen Frieden, die sich im zweiten Heft des ersten Bandes findet.

Der anonyme Rezensent ist bemüht, Kants Text als einen genuin deutschen Entwurf auszuflaggen, der zwar durchaus auf Reflexionen Rousseaus und des Abbé de Saint-Pierre zurückgreift, aber, da verfasst von einem »Original=Genie ${ }^{22}$, derlei Schützenhilfe gar nicht gebraucht hätte. Diese Abwehrhaltung gegen französische Einflüsse durchzieht auch einige der Beiträge Reichardts. In einem Text, der Schlegels Republikanismus-Aufsatz vorangestellt ist, beschwert sich Reichardt über die französisch-revolutionäre „Kürze des Stolzes wie die "Wortberedsamkeit der Schmeichelei« und fügt hinzu: „Der deutsche Charakter [...] ist der moralischen Freiheit [...] weit fähiger, als sein seit tausend Jahren verschränkter und verschrobener Nachbar mit unselig reizbaren Nerven und siedendem Blute. ${ }^{23}$ In der dezidierten Absetzung ${ }^{24}$ von den in den Peritexten sedimentierten chauvinistisch-agonalen Dispositiven der Zeitschrift gelangt nun Friedrich Schlegel zu einer Perspektive auf Kulturpolitik, die sich der Bedeutung des Massenmedialen bewusst wird. ${ }^{25}$ Denn in Schlegels Re-Evaluation des Republikanismus lassen sich $\mathrm{m}$. E. zwei Momente ausmachen, die sich in der Distributionspolitik des Heftes, die wesentlich von Johann Friedrich Unger geprägt ist, festgesetzt haben. Das erste Moment betrifft die Affirmation einer vorübergehenden Diktatur im republikanischen Staat, das zweite die zeittypische Perspektivierung des Staates wie des Menschen als eines 'Ganzen`. Ich werde daher

22 Deutschland (s. Anm. I9), I. Bd., 2. Stk., S. 267.

23 Ebd., 3. Bd., 7. Stk., S. 9.

24 In einem Brief vom 3I. Oktober 1797 an den Bruder August Wilhelm wird Schlegel Reichardt wie folgt charakterisieren: „Der Mann hat viel Gutes, aber da er nicht liberal ist, so würde es thöricht seyn, wenn ich mich entetiren wollte, in litterar.[ischer] Gemeinschaft mit ihm zu bleiben. Sein soidisant Republikanism politisch und litterarisch ist alter Aufklärungsberlinism, Opposizionsgeist gegen Obskuranten, und Franzosenhang, die er als Deutscher haßt und verachtet, ohne doch von ihnen lassen zu können, so wie er die Deutschen hinwiederum völlig wie ein Franzose verachtet [...] (KFSA 24, S. 30). Auch zuvor schon ist Schlegels Perspektive differenzierter als Reichardts gut dokumentierte Neigung zum Nationalhass.

25 Vgl. hierzu Ethel Matala de Mazza: Der verfasste Körper. Zum Projekt einer organischen Gemeinschaft in der Politischen Romantik. Freiburg 1999, S. $176 \mathrm{ff}$. 
kurz Schlegels Argumentation zusammenfassen und sie dann zwei Texten Johann Friedrich Ungers gegenüberstellen.

Wie erinnerlich universalisiert Schlegel Reichardts sdeutschen Gemeinsinn`zur `Gemeinschaft der Menschheit`. Sie konstituiert sich qua Kommunikation, die ganz im Sinne Rousseaus darauf abzielt einen allgemeinen Willen zu instituieren, indem sich die Individuen zu »eine[r] ununterbrochne[n] Masse $\ll^{26}$ veräußern. Schlegel erkennt nun ein Problem in der Differenz von allgemeinem Willen und "absolut allgemeinem (und also auch absolut beharrlichen) Wille[n] «, da letzterer »im Gebiete der Erfahrung nicht vorkommen kann, und nur in der Welt der reinen Gedanken existirt. « ${ }^{27}$ Für die künftige Republik bedeutet dies konsequenterweise, dass sie sich auf die »Fikzi o n« zu gründen hat,

einen empirischen Willen als Su r rogat des a priori gedachten absolut allgemeinen Willens gelten zu lassen; und da die reine Auflösung des politischen Problems unmöglich ist, sich mit der Approximation dieses praktischen $\mathrm{x}$ zu begnügen. ${ }^{28}$

An dieser Stelle greift Schlegel auf das Konzept der fictio juris zurück. Rechtsgültigkeit kann nur eine auf das Gesetz der Gleichheit gegründete Fiktion beanspruchen: »Der Wille d e r Mehrheit soll als Surrogat des allgemeinen Willens gelten. ${ }^{29}$ Folglich sedimentiert sich in diesem Willen die politische Macht. Diese Macht weist zwei Aggregatzustände auf; einen permanenten und einen transitorischen. Den transitorischen nennt Schlegel "Regierung", den permanenten nennt er »Konstitutzion $\aleph^{30}$. Hier nun tritt das Fiktionale im Sinne einer Metaphorisierung und imaginären Aufladung des republikanischen Staates in den Text wieder ein; der Staat wird präsentiert als Analogon zum Kantischen Denker: „Die konstitutiv e Macht entspricht der

26 Schlegel: „Versuch über den Begriff des Republikanismus« (s. Anm. I2), S. I9.

27 Ebd., S. 2I.

28 Ebd.

29 Ebd., S. 22.

30 Ebd., S. 26. 
Vernunft, die legislative dem Verstande, die richterliche der Urtheilskraft und die ex eku tive der Sinnlichkeit. « ${ }^{3 \mathrm{~S}}$ Schlegel macht sich mithin die Doppeldeutigkeit des Begriffes 'Konstitution zunutze, der Staats- aber auch Körperverfassung meinen kann. ${ }^{32}$ Damit ist zugleich angedeutet, dass die mit den Machtmomenten korrelierten Erkenntnismodule des Kantischen Denkers (sprich: des Menschen) nicht als für sich genommen einzelne, sondern als spezifische Relata interessieren:

Die Bestandtheile der politischen Macht verhalten sich untereinander und zu ihrem Ganzen, wie die verschiedenen Bestandtheile des Erkenntnisvermögens unter einander und zu ihrem Ganzen. ${ }^{33}$

Das Ganze des Erkenntnisvermögens, das Ganze der politischen Macht: zwei Mal integriert derselbe Begriff disparate Teilbereiche und macht sie vergleichbar. Er konvertiert den Denker/Menschen in den Staat und umgekehrt. Der Mensch kann in dieser Perspektive gar nicht anders als den republikanischen Staat zu wollen. Denkt man diese Konstellation weiter, dann wird klar, dass es nicht mehr als ein menschliches Individuum braucht, um einen Staat zu machen, denn schon dessen Erkenntnisvermögen entspricht schließlich der politischen Macht. Und tatsächlich liebäugelt Schlegel mit der Möglichkeit der diktatorischen Regierungsform. Diese Diktatur ist legitim, solange ihr Ende absehbar bleibt. Wie geht das zusammen mit Schlegels Bemerkung über die nähere Kennzeichnung der konstitutiven Macht, die, wie erinnerlich, der Vernunft entsprechen soll und von der es heißt,

3 Ebd.

32 Diese Volte halte ich für die eigentliche, originelle Leistung der Kant-Rezeption Friedrich Schlegels im Republikanismus-Aufsatz. Die historischen Interdependenzen von fictio juris und literarischer Fiktion in der Frühen Neuzeit thematisiert Luhmann: "Literatur als fiktionale Realität« (s. Anm. I7), an dessen Perspektivierung ich mich anschließe. Für eine Analyse der politischen Potenziale der Körper-Metaphorik vgl. Albrecht Koschorke/Susanne Lüdemann u.a.: Der fiktive Staat. Konstruktionen des politischen Körpers in der Geschichte Europas. Frankfurt a. M. 2007.

33 Schlegel: "Versuch über den Begriff des Republikanismus" (s. Anm. I2), S. 26. 
sie sei "n o thwendig diktato ris ch «?34 Auch sie kann nur vorübergehend alleine >regieren`, es bedarf schließlich eines "A k tes der Akzep tati o n«, 35 der ihre Vorgaben nachträglich ratifiziert. Die konstitutive Macht wird dergestalt ins Ganze re-implementiert, sie ist fortan kein exzeptioneller Teil mehr. Schlegels Konzeption orientiert sich mithin an einem dynamischen Elitarismus, in dem sich eine kleine Gruppe oder ein Einzelner zum intellektuellen Oberhaupt aufwirft, um sich dann mit der Masse, die nun gleichfalls dem Ziel der intellektuellen Vervollkommnung näher gekommen ist, wiederzuvereinigen. Nimmt man nun die romantischen Phantasmen der kritischen Diktatur wie die Auffassung, der Leser sei noch zu konstruieren, ernst, so ließe sich argumentieren, dass die Mitarbeiter des Journals, der Herausgeber Reichardt, der kulturpolitische Beiträger Friedrich Schlegel wie der Verleger und Buchdrucker Unger Deutschland selbst als Teil dieser Elite verstehen, als Kooperation des Herausgebers Reichardt, des Verlegers Unger, des ständigen Beiträgers Friedrich Schlegel und einiger anderer Intellektueller. Die Argumentation liefert also eine Begründung für die Legitimität der publikatorischen Operationen, für die Angemessenheit der translatio und des agon, die nur vorübergehend den Ton der Zeitschrift bestimmen werden, und sie hat den verlegerischen Erfolg im Blick: die `Akzeptation der hier gedruckten Ideen ist ihr Abonnement; es unterliegt den folgenden von Unger aufgestellten Bestimmungen:

Von diesem Journal wird [...], in der Mitte jedes Monats, ein Stück von 7 bis 8 Bogen erscheinen; der Jahrgang kostet Fünf Thaler Preuß. Courant. Das hiesige königliche Hofpostamt hat die Hauptversendung übernommen, daß folglich ein jeder, der an Orten wohnt wo keine Buchhandlung ist, sich an das nächstgelegene Postamt deshalb wenden kann, und dies Journal regelmäßig erhalten wird. ${ }^{36}$

Universalisierte Kommunikation durch optimierte Distribution: Jeder im zukünftigen republikanischen Staat kann die Zeit-

34 Ebd.

35 Ebd.

36 Deutschland (s. Anm. 19), S. 5. 
schrift beziehen, die Allianz mit der um I8oo fortschrittlichsten Nachrichtentechnologie, der Post, sichert die Übertragung der konstitutiven Diktate. Dabei machen sich die Abonnenten »a uf ein ganzes Jahr verbindlich", wobei »[e]inzelne Stücke nicht verkauft ${ }^{37}$ werden, die Zeitschrift also nur als Ganze zu beziehen ist. Das ist nicht bloß eine kluge ökonomische Entscheidung, sondern Teil des Programms. Insofern sind Schlegels elitaristische Rechtfertigungen einer Diktatur, die durch notwendige Fiktionen abgesichert ist, selbstreflexiv und typisch romantisch, in ihnen wird eine Poetik (des Journals) Deutschland(s) greifbar.

Die Verschränkung von Ganzheitsvorstellungen und transitorischer Diktatur hinterlassen nun aber auch - mit gewissem zeitlichen Abstand - ihre Spuren in jener Organisationseinheit, die Druck, Verlag und Ausschickung des Journals besorgt. Im Jahr I802 gibt Johann Friedrich Unger seiner Offizin eine Verfassung. Der Akt gründet in einem Reformbegehren; als gewiefter Historiker des Buchdrucks beobachtet Unger gegen Ende des i8. Jahrhunderts eine gestiegene Anspruchshaltung auf Seiten literarischer Akteure. Die literarischen Nationaldiskurse haben die Erwartungen an die Druck- und Ausstattungsleistung der Offizin erhöht, was sich wiederum in einer Professionalisierungserwartung an die innerinstitutionellen Standards ausdrückt: „Die Buchdruckerei ist eine so edle und nützliche Kunst, daß man bei denen, welche sie ausüben, einen gewissen Grad von Kultur voraussetzen sollte. (38 $^{8}$ Der Ton dieses Eingangssatzes aber gibt zu verstehen, dass die Kultur gerade nicht vorausgesetzt werden kann, sondern erst entstehen muss, womit die Wirkabsicht der Verfassung denn auch identifiziert ist. Voraussetzung einer Wirkung ist, nicht anders als in den Theorien des Gemeinwesens von Rousseau, Kant und Friedrich Schlegel, ein Imperativ der Bildung des Einzelnen. Interessant ist nun, wie Unger seine `Gesellschaft ‘ perspektiviert. Im Zentrum der neuen Verfassung der Offizin steht ein Konzept, das viele der bei Unger gedruckten Texte Schlegels durchzieht und

37 Ebd., S. 6.

38 Innere Verfassung der Ungerschen Buchdruckerei. Anerkannt von sämmtlichen Mitgliedern derselben. Berlin I802, S. 5. 
Es kann seyn, daß nachstehende Verfügungen Manchem bei der ersten Ansicht zu streng vorkommen möchten; wer aber mit richtigem Sinn das Ganze übersieht, und gern das Gute befördern hilft, wird den Nutzen dieser anscheinenden Strenge nicht verkennen, und die Ordnung und Ruhe, womit er in meiner Offizin die Stunden der Arbeit zubringen kann, wird ihn, wenn alles schön in einander greift, für manche kleine Aufopferung reichlich belohnen. Ich muß bitten, meine Absicht bei diesen Anordnungen vorurtheilfrei zu überlegen, und sich nicht durch Eingebungen einiger übelgesinnter Mitglieder dagegen verstimmen zu lassen. Dadurch würde alles, was zum Besten des Ganzen und der allgemeinen Zufriedenheit bewirkt werden könnte, gänzlich vereitelt. ${ }^{9}$

Was ist das Ganze, zu dessen Besten die Verfassung erlassen wird? Ein Blick in ihre Präambel hilft weiter. »Immer war es mein eifriger Wunsch«, schreibt Unger,

Ordnung und Sittlichkeit wieder herzustellen, und diese an sich so ehrwürdige Kunst zu der Höhe der Achtung, auf welcher sie noch lange nach ihrem Entstehen stand, zurückzuführen. Diesem gewiß billigen Wunsche nachzukommen, habe ich, da die Buchdrucker nicht handwerksmäßig zünftig sind, und jede Buchdruckerei für sich ein Ganzes ausmacht, nachstehende Ordnung für meine Druckerei entworfen. ${ }^{4 \circ}$

Die Buchdruckerei ist ein `Ganzes`, eine korporative und kooperative Einheit, keine Summe aus zünftig organisierten Druckern und Setzern. Eine solche Institution braucht eigene Reglements und d. h. Fiktionen, die sie konstituieren. Vor dem Hintergrund des Wissens um die politisch-ästhetische Validierung des Konzepts des 'Ganzen im von Unger gedruckten Republikanismus-Aufsatz Friedrich Schlegels lautet meine These: Unger imaginiert seine Offizin als Republik und sich als deren Vorsteher, der konstitutive Diktate ausgibt. Diese Verfassung wird publiziert, sie kann prinzi-

39 Ebd., S. I4.

40 Ebd., S. 5 . 
piell von jedem gelesen werden, der einen Preis von 4 Groschen zu

entrichten bereit ist. Sie soll anderen Offizinen als Muster dienen, beansprucht also womöglich universale Gültigkeit. Diese Gültigkeit selbst aber wird nicht durch apodiktische Weisung zu legitimieren versucht auch Unger begreift seine Diktatur nur als transitorischen Zustand. Der letzte Absatz, der durch eine Zierleiste vom übrigen Verfassungstext getrennt ist, präsentiert die republikanische (Neu-) Gründungsszene des typographischen Staates:

\begin{abstract}
Als alle diese Punkte verlesen, gehörig auseinander gesetzt und von den sämmtlichen Mitgliedern meiner Druckerei geprüft waren, stimmte die ganze Gesellschaft meinem Entwurfe zur besseren Ordnung bei. Sie sahen zu meiner großen Freude alle die guten Folgen ein [...]. Wie sehr danke ich es dieser meiner Gesellschaft im Namen unserer Kunst, daß sie so einsichtsvoll und unbefangen meine Vorschläge annahm, und daß sie den ersten Schritt that, unsere Kunst wieder zu Ehren zu bringen. ${ }^{41}$
\end{abstract}

Die "ganze Gesellschaft«, bestehend aus »sämmtlichen Mitgliedern«, äußert den allgemeinen Willen und gibt sich die Verfassung. Unger war bloß intellektueller Vorreiter, der die allen potenziell inhärenten Gedanken zu Papier brachte. Die Etablierung einer typographischen Kultur ist möglich, ihren Antrieb bezieht sie aus den Imaginationen romantischen Denkens, das den republikanischen Staat über das Konzept des Ganzen mit dem bildungsfähigen Individuum verknüpft.

In Theodore Ziolkowskis Studie zur deutschen Romantik und ihren Institutionen werden das Bergwerk, das Recht, die Universität, das Irrenhaus und das Museum thematisch, die Druckerei wird es nicht. Anknüpfend an die Ergebnisse der soziologischen Institutionenforschung Talcott Parsons', Shmuel N. Eisenstadts und Bronislav Malinowskis fasst Ziolkwoski Institutionen als Bedürfniskatalysatoren. So sedimentiert sich

4I Ebd., S. $28 \mathrm{f}$. 
das Bedürfnis [...] nach Gerechtigkeit in der Institution des Rechts [...], das Bedürfnis nach Bildung in der Institution der Universität, das Bedürfnis nach Gesundheit in der Institution der Nervenheilanstalt, das Bedürfnis nach Kultur in der Institution des Museums und das Bedürfnis nach bestimmten Rohstoffen in der Institution des Bergbaus. ${ }^{42}$

Dabei geht es Ziolkowski "um die Wechselwirkung zwischen konkreten literarischen Werken und der Gesellschaft. «" wenn man den letztlich opak bleibenden Mechanismus der $\mathrm{Zu}$ ordnung von gesellschaftlichen Bedürfnissen und Institutionen als Erklärung akzeptiert, wird nicht klar, warum etwa ein derart ubiquitär-abstraktes Bedürfnis wie dasjenige nach Kultur mit dem Bedürfnis nach bestimmten Rohstoffen auf einer Stufe steht und warum es seine Veräußerung ausgerechnet in der unwahrscheinlichen Institution Museum erfahren soll, nicht aber auch in den Institutionen Theater, Salon oder Leihbibliothek. Das Zuordnungsproblem ergibt sich aus Ziolkowskis gut begründeter thematologischer Perspektive: Das Bergwerk ist ein Motiv oder Thema der romantischen Literatur, genauso wie das Recht, die Nervenheilanstalt, das Museum und die Universität. Zugleich spielen die Institutionen eine Rolle im Leben der romantischen Autoren, sodass sich mit der Textinterpretation eine biographische Sicht verschränken lässt. Dass die Druckerei zu den klassizistischromantischen Institutionen gehört, dass sie eine Art Superinstitution bildet (wenngleich sie - vielleicht gerade deshalb - nie zu einem bedeutenden Motiv fiktionaler romantischer Literatur geworden ist ${ }^{44}$ ), muss demgegenüber nicht betont werden. Die meisten der literaturhistorisch relevanten Texte der Epoche liegen - bei aller Wichtigkeit handschriftlicher Zirkulation um I80o in gedruckter Form vor, Verhandlungen mit Verlegern gehören zum Alltagsgeschäft der Autoren, welchen Berufen auch sonst

42 Theodore Ziolkowski: Das Amt der Poeten. Die deutsche Romantik und ihre Institutionen. Übers. von Lothar Müller. München 1994, S. 20.

43 Ebd., S. I9.

44 Ausnahmen bestätigen bekanntlich nur die Regel, vgl. etwa Jean Pauls Siebenkäs (1796 f.) oder auch Leben Fibels, Verfasser der Bienrodischen Fibel (I8I2). 
sie nachgegangen sein mögen. Manchmal ergeben sich daraus spannungsgeladene Konstellationen, wie Hannelore Schlaffer am Beispiel Clemens Brentanos gezeigt hat. ${ }^{45}$ Daher wurde hier versucht, der intrinsischen Verschränkung von romantischer Literatur und Gesellschaft nachzuspüren, indem nach der formativen Potenz der romantischen Literatur im Kontext der Institutionen und Medien gefragt wurde, welche nun ihrerseits selbst an der Materialisation von Literatur beteiligt sind.

Derlei Versuche der Verschaltung von Text und Kontext sehen sich für gewöhnlich mit der Frage konfrontiert, 'wie weit man gehen darf.$^{46} \mathrm{Um}$ Missverständnissen vorzubeugen: Es geht nicht um die Behauptung starker Kausalitäten - dass Unger Schlegels Republikanismus-Aufsatz im Vorfeld seiner Reformbemühungen erneut gelesen hätte, lässt sich nicht belegen. Wohl aber lässt sich sagen, dass die Gleichzeitigkeit von realweltlicher, distributiver Konstellation Schlegel/Reichardt/Unger und politischer Rhetorik im Republikanismus-Aufsatz wie in Ungers Verfassung eine Beschreibung verdient. Denn wenn Friedrich Schlegels Republikanismus sich in der Distributionspolitik des Journals Deutschland reflektiert findet und Johann Friedrich Unger in Korrelation zu Schlegels republikanischem Ganzheitskonzept seiner Offizin eine Verfassung 'diktiert`, dann lässt sich von einer Allianz romantischer Ideen mit sozialen Formationen sprechen, die die Behauptung, romantische Politik habe kein empirisch-gesellschaftliches Korrelat, fragwürdig werden lässt.

45 Hannelore Schlaffer: „Gitarre und Druckerei. Clemens Brentanos Schwierigkeiten beim Publizieren«. In: Klaus Grubmüller/Günter Hess (Hg.): Bildungsexklusivität und volkssprachliche Literatur/Literatur vor Lessing - nur für Experten. Tübingen 1986, S. 5I-58.

46 Vgl. Moritz Baßler: Die kulturpoetische Funktion und das Archiv. Eine literaturwissenschaftliche Text-Kontext-Theorie. Tübingen/Basel 2005, S. 79 f. Baßler, der sich für eine theoretische Fundierung des New Historicism interessiert, macht das strukturalistische Konzept der Ko-Okkurrenz stark, um die mitunter staunen machende Gleichzeitigkeit textueller Bestände beschreiben zu können. Das Konzept des Ganzen bei Unger wie bei Schlegel stünden in einem solchen ko-okkurrenten Verhältnis zueinander. Allerdings wäre aus der hier veranschlagten Perspektive zu ergänzen, dass diese Konzepte wieder in die Realwelt hineinreichen und tatsächliche Folgen im Sozialen zeitigen. 
264 Es wäre vielleicht nicht wenig erreicht, wenn die hier angestellten, durchaus vorläufigen Überlegungen dazu anregen könnten, die Praxis der romantischen Medienpolitik schärfer ins Auge zu fassen, als das bisher geschehen ist. Für ein besseres Verständnis der Kulturpolitik der Romantik ist dieses Vorhaben allemal von hoher Relevanz. Denn die Durchsetzung kulturkonstitutiver und d.h. immer auch exkludierender Unterscheidungen ist auf Machtkapazitäten angewiesen. Fragt man danach, wie diese erzeugt werden, so fragt man unausweichlich nach den (Massen-) Medien romantischer Kulturpolitik. 